\title{
Thermal Unit Commitment Using Binary Differential Evolution
}

\author{
Yun-Won Jeong*, Woo-Nam Lee**, Hyun-Houng Kim**, \\ Jong-Bae Park ${ }^{\dagger}$ and Joong-Rin Shin**
}

\begin{abstract}
This paper presents a new approach for thermal unit commitment (UC) using a differential evolution (DE) algorithm. DE is an effective, robust, and simple global optimization algorithm which only has a few control parameters and has been successfully applied to a wide range of optimization problems. However, the standard DE cannot be applied to binary optimization problems such as UC problems since it is restricted to continuous-valued spaces. This paper proposes binary differential evolution (BDE), which enables the DE to operate in binary spaces and applies the proposed BDE to UC problems. Furthermore, this paper includes heuristic-based constraint treatment techniques to deal with the minimum up/down time and spinning reserve constraints in UC problems. Since excessive spinning reserves can incur high operation costs, the unit de-commitment strategy is also introduced to improve the solution quality. To demonstrate the performance of the proposed BDE, it is applied to largescale power systems of up to 100-units with a 24-hour demand horizon.
\end{abstract}

Keywords: Combinatorial optimization, unit commitment, binary differential evolution, constrainthandling

\section{Introduction}

Unit commitment (UC) involves scheduling the on/off states of generating units to minimize the operating cost for a given time horizon. The committed units must meet the system's forecasted demand and spinning reserve requirement at minimum operating cost, subject to a large set of operating constraints. The UC problem, one of the most important tasks in short-term operation planning of modern power systems, has a significant influence on the secure and economic operation of power systems [1]. Optimal commitment scheduling cannot only save millions of dollars for power companies, it also ensures system reliability by maintaining the proper spinning reserve. The UC problem is mathematically formulated as a nonlinear, largescale and mixed integer combinatorial optimization problem [2-16]. The number of combinations of $0-1$ variables grows exponentially for a large-scale UC problem. Therefore, the UC is one of the most difficult problems in the area of power system optimization.

Over the past decades, many salient optimization methods have been developed to solve the UC problem. The exact solution to the problem can be obtained only by complete enumeration, which cannot be applied to realistic power due to its excessive computation time requirements [1]. Research efforts, therefore, have concentrated on efficient and near-optimal UC algorithms which can be applied to realistic power systems and have reasonable storage and

$\dagger \quad$ Corresponding Author: Dept. of Electrical Engineering, Konkuk University, Korea. (jbaepark@konkuk.ac.kr)

* Business Development Group, POSCO POWER Corp., Korea. (ywjeong@poscopower.co.kr)

** Dept. of Electrical Engineering, Konkuk University, Korea. (\{mansmind, hhblue, jrshin\}@konkuk.ac.kr)

Received 26 February 2009; Accepted 7 July 2009 computation time requirements. The optimization methods for UC problems can be divided into two classes through a survey of literature as follows: The first are numerical optimization techniques such as priority list methods [2,3], dynamic programming [4,5], Lagrangian relaxation methods [6,7], branch-and-bound methods [8], and mixedinteger programming [9]. The other are stochastic search methods such as genetic algorithms (GA) [10,11], evolutionary programming (EP) [12,13], simulated annealing (SA) [14,15], and particle swarm optimization (PSO) [16].

Differential evolution (DE) developed by Storn and Price [17] is a simple yet powerful heuristic optimizer for solving nonlinear, non-differentiable, and multi-modal optimization problems. In DE, the fitness of an offspring competes one-to-one with that of the corresponding parent. This one-to-one competition, which is different from other evolutionary algorithms (EAs), gives rise to a faster convergence rate. In addition, DE has a limited number of parameters, which include mutation factor, crossover rate and population size, in comparison with other competing heuristic optimization methods [18].

This paper presents a new DE-based approach for solving UC problems. Many optimization problems, including UC problems, are set in a space featuring discrete, qualitative distinctions between variables and between levels of variables. However, the standard DE algorithms have operated in only continuous spaces. This paper proposes the binary version of the $\mathrm{DE}$ algorithm, called binary differential evolution (BDE), to find the optimal solution in binary optimization problems and applies the proposed BDE to UC problems. The binary version of DE enables the DE to operate in binary spaces. The structure of the algorithm is effectively the same as the standard DE. The difference of the $\mathrm{BDE}$, compared to the standard $\mathrm{DE}$, is that the individ- 
ual's chromosome is a binary vector.

In addition, to effectively satisfy the minimum up/down time and spinning reserve constraints in UC problems, heuristic-based constraint treatment techniques are proposed in order to improve the solution quality without causing a decline in computational efficiency. To prevent high operating costs due to excessive spinning reserves, the unit decommitment approach is also proposed. To demonstrate the performance of the proposed BDE, test systems of up to 100-units along with 24-hour load demands are tested and its results are compared with those of previous works.

\section{Formulation of Unit Commitment}

\subsection{Objective Function}

The main objective of the UC problem is to minimize the total operating cost of generating units during the scheduling horizon, subject to a number of system and unit constraints [1]. The overall objective function of the UC problem is expressed as the sum of fuel, start-up, and shutdown costs of generating units.

\section{1) Fuel Cost Function}

For all committed generating units, the total fuel cost is minimized by economically dispatching the units. The fuel cost function of unit $i$ at hour $t$ is expressed as a second order polynomial as follows:

$$
F_{i}\left(P_{i, t}\right)=a_{i}+b_{i} P_{i, t}+c_{i} P_{i, t}^{2}
$$

where $P_{i, t}$ is the power generation of unit $i$ at hour $t$ and $a_{i}, b_{i}, c_{i}$ are the cost coefficients of unit $i$.

\section{2) Start-Up Costs}

Start-up costs for restarting a decommitted thermal unit, which is related to the temperature of the boiler, is included in the model. That is, the start-up cost depends on the number of hours during which the unit has been off. Startup costs will be a high cold cost $\left(S U_{C, i}\right.$ ) when down time duration exceeds the cold start hour $\left(T_{\text {cold }, i}\right)$ in excess of minimum down time and will be a low hot cost $\left(S U_{H, i}\right)$ when down time duration does not exceed the cold start hour in excess of minimum down times. In general, the start-up cost is described in the two-step function as follows:

$$
S U_{i, t}= \begin{cases}S U_{H, i} & \text { if } \mathrm{MDT}_{i} \leq \mathrm{TOFF}_{i, t} \leq M D T_{i}+T_{\text {cold }, i} \\ S U_{C, i} & \text { if } \mathrm{TOFF}_{i, t}>\mathrm{MDT}_{i}+T_{\text {cold }, i}\end{cases}
$$

where $T O F F_{i, t}$ is the duration for which unit $i$ is continuously off-line at hour $t$ and $M D T_{i}$ is the minimum down-time of unit $i$.

\section{3) Shut-Down Costs}

Shut-down costs are usually modeled as a constant value for each unit per shutdown. In this paper, the shutdown costs have been taken as equal to 0 for all units, and is excluded from the objective function.

Consequently, the UC objective function is given by the minimization of the following cost function:

$$
\min \sum_{t=1}^{T} \sum_{i=1}^{N}\left[F_{i}\left(P_{i, t}\right) \alpha_{i, t}+S U_{i, t}\left(1-\alpha_{i, t-1}\right) \alpha_{i, t}\right]
$$

where $T$ is the number of scheduling periods, $N$ is the number of generating units, and $\alpha_{i, t}$ is the on/off status of unit $i$ at hour $t$. $\alpha_{i, t}$ is set to be 1 when unit $i$ is online, and $\alpha_{i, t}$ is set to be 0 when unit $i$ is off-line.

\subsection{System Constraints}

\section{1) Load Balance Constraints}

The sum of the unit generation output at each hour must satisfy the system load demand requirement of the corresponding hour as follows:

$$
\sum_{t=1}^{N} P_{i, t} \cdot \alpha_{i, t}=P D_{t}
$$

where $P D_{t}$ is the total system demand at hour $t$.

\section{2) Spinning Reserve Constraints}

Spinning reserves must be available during the operation of a power system in order to minimize the probability of load interruption. The reserve is considered to be a prespecified amount or a given percentage of the forecasted peak demand. Spinning reserves can be specified in terms of excess megawatt capacity, which is expressed by

$$
\sum_{t=1}^{N} P_{i, \max } \cdot \alpha_{i, t} \geq P D_{t}+S R_{t}
$$

where $S R_{t}$ is the required spinning reserve at hour $t$.

\section{3) Generation Limit Constraints}

The power produced by each unit must be within certain limits, as indicated below:

$$
\alpha_{i, t} P_{i, \min } \leq P_{i, t} \leq \alpha_{i, t} P_{i, \max }
$$

where $P_{i, \min }$ and $P_{i, \text { max }}$ are the minimum and maximum generation limits of unit $i$, respectively.

\section{4) Generation Ramping Constraints}

Due to the unit mechanical and thermal stress limitations, the ramp-up and ramp-down constraints are given below:

$$
R D_{i} \leq P_{i, t}-P_{i,(t-1)} \leq R U_{i}
$$

where $R D_{i}$ and $R U_{i}$ are the ramp-down and ramp-up limits of unit $i$, respectively.

\section{5) Minimum Up-Time/Down-Time Constraints}

The unit cannot be turned off immediately once it is 
committed, and vice versa. The minimum up/down time constraints indicate that a unit must be on/off for a certain number of hours before it can be shut off or brought online, respectively. These constraints are given by

$$
\begin{aligned}
\mathrm{TON}_{i, t} & \geq \mathrm{MUT}_{i} \\
\mathrm{TOFF}_{i, t} & \geq M D T_{i}
\end{aligned}
$$

where $\mathrm{TON}_{i, t}$ is the duration for which unit $i$ is continuously on-line at hour $t$ and $M U T_{i}$ is the minimum up-time of unit $i$.

\section{BDE for UC Problems}

\subsection{Overview of DE}

A DE developed by Storn and Price [17] is a population-based evolutionary computation technique. Due to its simple but powerful and straightforward features, it is very attractive for resolving global optimization problems. In $\mathrm{DE}$, the fitness of an offspring competes one-to-one with that of the corresponding parent. This one-to-one competition will give rise to a faster convergence rate than other EAs. In addition, only a few control parameters are required in comparison with other competing heuristic optimization methods [18]. The basic algorithm of DE typically consists of four phases: 1) initialization, 2) mutation, 3) crossover, and 4) selection phases. The mutation and crossover are used to generate new individuals, and selection then determines that the individuals will survive into the next generation. The performance of a DE algorithm usually depends on three parameters, i.e., population size, mutation factor, and crossover rate [17], [18]. The procedure of DE can be summarized as the following pseudocode:

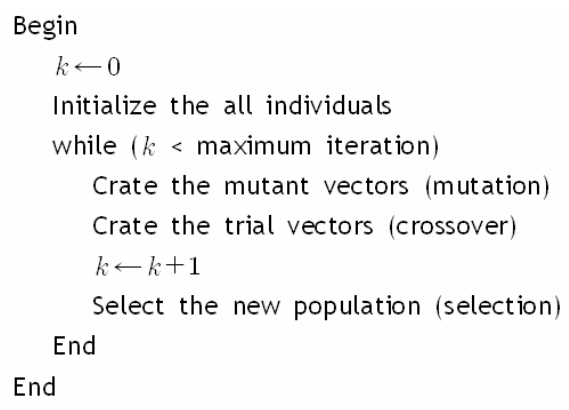

Fig. 1. Pseudocode of the DE

\subsection{Implementation of BDE for UC Problems}

Since the UC in power systems involves determining the on/off states of generating units that minimize the operating cost for a given time horizon, the decision variables are the on/off status of generating units. After determining the optimal combination of commitment scheduling, the optimal power outputs of the committed units are determined through an economic dispatch (ED) procedure. Since the fuel cost function of a generating unit is approximately represented as a quadratic function as described in (1), the ED problem can be solved by numerical techniques.

For searching in parallel as with other evolutionary algorithms, a population of individuals at the $k$ th generation is represented as $X(k)=\left\{X_{1}(k), X_{2}(k), \cdots, \quad X_{N P}(k)\right\}$, where NP is the population size. Here, the $i$ th individual is represented as the binary target vector $X_{i}(k)=\left\{x_{i 1}(k), \cdots, x_{i N}(k)\right\} \quad, \quad i \in[1, N P]$, which is formed by selecting either 0 or 1 for each bit. In the subsequent sections, the detailed procedures of BDE for scheduling the on/off states of units are described.

\section{1) Initialization of population}

In the initialization process, the initial individuals are randomly chosen. After generating a random number $r_{i j}$ between 0 and 1 , an initial value of the $j$ th element in the ith individual (i.e., $\left.x_{i j}(0)\right)$ takes a value of 1 if $r_{i j}$ is less than $1 / 2$, otherwise $x_{i j}(0)$ set to be 0 .

\section{2) Mutation operation}

A mutant vector $U_{i}(k)=\left\{u_{i 1}(k), u_{i 2}(k) \cdots, u_{i N}(k)\right\}$ is generated based on the present individual $X_{i}(k)$ as follows:

$$
U_{i}(k)=\operatorname{sign}\left[X_{r 1}(k)+M F \times\left(X_{r 2}(k)-X_{r 3}(k)\right)\right]
$$
with random indices $r_{1}, r_{2}, r_{3} \in[1, N P]$. Note that the randomly chosen integers $r_{1}, r_{2}$ and $r_{3}$ are have to be different from each other and from the running index $i$ (i.e. $i \neq r_{1} \neq r_{2} \neq r_{3}$, ), so that $N P$ must be at least four. $M F$ is the mutation factor. In the standard DE, $M F$ is a real and constant factor, which controls the amplification of the difference between two individuals and is usually taken from the range $[0,1]$. In the proposed BDE, however, $M F$ is set as 1.0 since each element in the individuals takes a value of 0 or 1 . The sign function in (10) is defined by:

$$
\operatorname{sign}[\eta]= \begin{cases}0 & \text { if } \eta \leq 0 \\ 1 & \text { if } \eta \geq 1\end{cases}
$$

\section{3) Crossover operation}

In order to increase the diversity of the population, crossover is introduced. The trial vector $V_{i}(k)=\left\{v_{i 1}(k), v_{i 2}(k) \cdots, v_{i N}(k)\right\}$ is generated as follows:

$$
v_{i j}(k)= \begin{cases}v_{i j}(k) & \text { if } r_{i j} \leq C R \\ x_{i j}(k) & \text { otherwise }\end{cases}
$$

for $j=1,2, \ldots, N$. $C R$ is the crossover rate in the range $[0,1]$.

\section{4) Selection operation}

To create the new population in the next generation $k+1$, the fitness value of the trial vector $V_{i}(k)$ is compared with its parent vector $X_{i}(k)$. If the trial vector 
$V_{i}(k)$ yields a smaller cost function value than $X_{i}(k)$, then $X_{i}(k+1)$ is set to $V_{i}(k)$. Otherwise, the target vector $X_{i}(k)$ is retained.

$$
X_{i}(k+1)=\left\{\begin{array}{l}
V_{i}(k) \text { if } f\left(V_{i}(k)\right) \leq f\left(X_{i}(k)\right) \\
X_{i}(k) \text { otherwise }
\end{array}\right.
$$

\section{5) Stopping Criteria}

The proposed algorithm is terminated if the iteration reaches a pre-specified maximum iteration.

\subsection{Constraints Treatment Method}

Penalty functions are the most popular methods in AIbased optimization techniques to handle the system constraints due to their simple concept and convenience to implement. However, these methods have certain weaknesses as the penalty functions tend to be ill-behaved near the boundary of the feasible region when the penalty parameters are large [19]. To overcome these penalty function drawbacks, therefore, this paper proposes efficient heuristic-based constraint treatment methods.

It is very important to create a population satisfying the constraints when solving UC problems. This paper proposes constraint-handling techniques for the minimum up/down time and the spinning reserve constraints. In the evolutionary process for solving UC problems, random bits flipping of state variables occurs, thereby the constraints may be frequently violated. In this paper, therefore, heuristic-based repair algorithms are proposed to accelerate the solution quality and to avoid generating infeasible solutions. To reduce the operating costs incurred by the excessive spinning reserve, the unit de-commitment approach is also proposed.

\section{1) Minimum Up-Time and Down-Time Constraints}

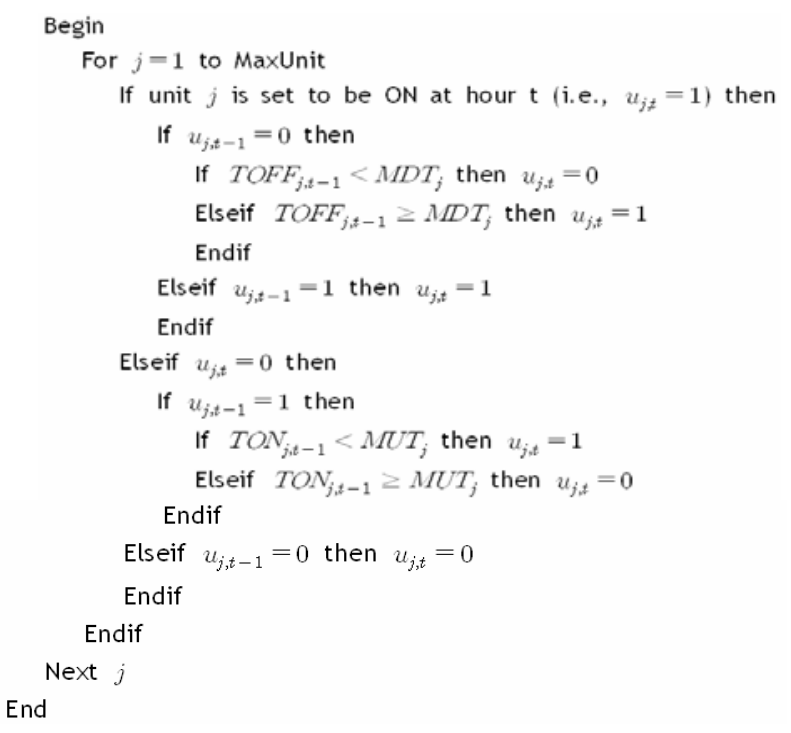

Fig. 2. Pseudocode for handling the minimum up-time and down-time constraints
When modifying the position of each particle, the minimum up/down time constraints should be satisfied. To do this, this paper proposes a heuristic-based constraint treatment technique as illustrated in the pseudo-code below.

\section{2) Spinning Reserve Constraint}

Adequate spinning reserves are required to maintain the system's reliability for a given time horizon. If the spinning reserve constraint is violated, the system suffers from a deficiency in its units. This paper proposes an efficient heuristic-based repair method which is launched when the spinning reserve is deficient at any scheduling period in order to avoid generating infeasible solutions. In the proposed repair process, de-committed units are forced to turn on until the spinning reserve constraint is satisfied as shown in Fig. 3.

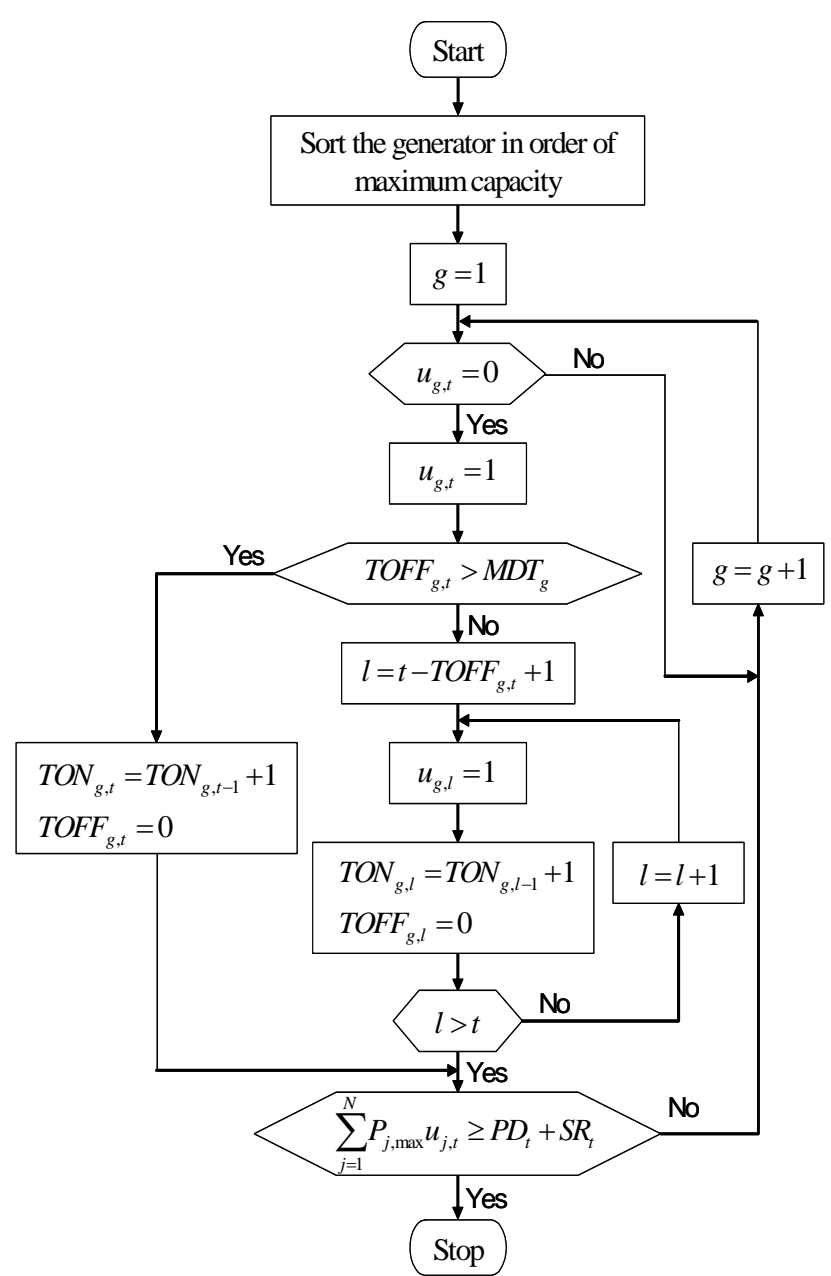

Fig. 3. Flowchart of repair algorithm for handling spinning reserve constraint

\section{3) Unit De-commitment for Excessive Spinning Reserve}

An excessive spinning reserve is not desirable due to the high operation cost. Therefore, this paper proposes a heuristic-based unit de-commitment process to reduce the excessive spinning reserve, leading to cost savings, as illustrated in Fig. 4. 


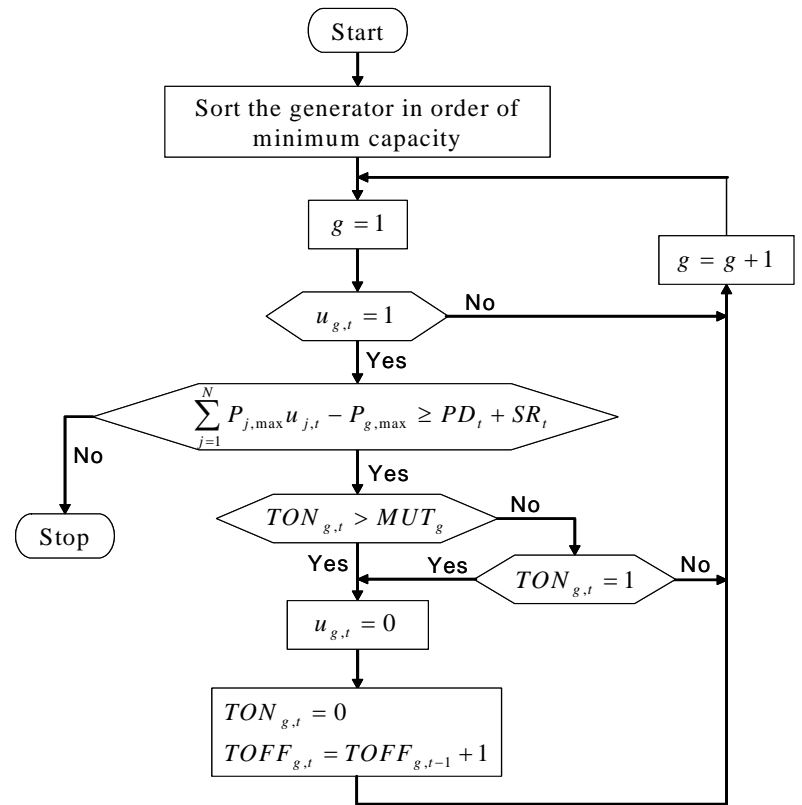

Fig. 4. Flowchart of unit de-commitment for prevention of excess spinning reserve

\section{Case Studies}

The proposed BDE is initially tested on a system of 10 generating units along with a 24-hour time horizon. The unit characteristics of the base 10-unit system and the demand are given in [10]. Subsequently, the 20, 40, 60, 80, and 100 unit data are obtained by duplicating the base case (10 units), and the load demands are adjusted in proportion to the system size. In all cases, the spinning reserve requirements are assumed to be $10 \%$ of the hourly demand. For each test case, 50 independent trials are conducted to compare the solution quality and convergence characteristics. Numerical tests have been executed on a Pentium VI 2.0GHz computer.

In implementing the proposed algorithms, some parameters must be determined in advance. In this paper the parameters were set through experiments as follows:

- Population size $N P=20$;

- Maximum iteration count iter $_{\max }=1,000$;

- Crossover rate $C R=0.1$.

The best, average, worst costs, and standard deviation for the test systems achieved by the proposed BDE algorithm are summarized in Table 1.

Table 1. Simulation results of the proposed BDE for the test systems

\begin{tabular}{ccccc}
\hline Units & Best Cost (\$) & Average Cost (\$) & Worst Cost (\$) & $\begin{array}{c}\text { Standard } \\
\text { Deviation }\end{array}$ \\
\hline 10 & 563,997 & 563,997 & 563,997 & 0 \\
20 & $1,123,998$ & $1,124,374$ & $1,124,927$ & 217.79 \\
40 & $2,245,700$ & $2,246,600$ & $2,247,284$ & 361.77 \\
60 & $3,367,066$ & $3,367,405$ & $3,367,783$ & 244.55 \\
80 & $4,489,022$ & $4,490,456$ & $4,491,262$ & 533.74 \\
100 & $5,609,341$ & $5,609,984$ & $5,610,608$ & 346.73 \\
\hline
\end{tabular}

In Table 2, the best results of the BDE are compared with those of Lagrange relaxation (LR) [10], genetic algorithm (GA) [10], evolutionary programming (EP) [12], simulated annealing (SA) [15], and improved particle swarm optimization (IPSO) [16]. Table 2 reveals that the proposed BDE is obviously superior to the existing methods although it couldn't obtain a better solution than the IPSO for the 10-unit system.

Table 2. Comparison of best results of each method

\begin{tabular}{ccccccc}
\hline Method & 10 -unit & 20-unit & 40-unit & 60-unit & 80-unit & 100 -unit \\
\hline LR [10] & 565,825 & $1,130,660$ & $2,258,503$ & $3,394,066$ & $4,526,022$ & $5,657,277$ \\
GA [10] & 565,825 & $1,126,243$ & $2,251,911$ & $3,376,625$ & $4,504,933$ & $5,627,437$ \\
EP [12] & 564,551 & $1,125,494$ & $2,249,093$ & $3,371,611$ & $4,498,479$ & $5,623,885$ \\
SA [15] & 565,828 & $1,126,251$ & $2,250,063$ & N/A & $4,498,076$ & $5,617,876$ \\
IPSO[16] & $\mathbf{5 6 3 , 9 5 4}$ & $1,125,279$ & $2,248,163$ & $3,370,979$ & $4,495,032$ & $5,619,284$ \\
BDE & 563,977 & $\mathbf{1 , 1 2 3 , 9 9 8}$ & $\mathbf{2 , 2 4 5 , 7 0 0}$ & $\mathbf{3 , 3 6 7 , 0 6 6}$ & $\mathbf{4 , 4 8 9 , 0 2 2}$ & $\mathbf{5 , 6 0 9 , 3 4 1}$ \\
\hline
\end{tabular}

For the 10-unit and 100-unit systems, the commitment schedules during a planning horizon obtained by the proposed BDE are described in Table 3 and Table 4, respectively.

Table 3. Unit scheduling and corresponding cost for 10unit system

\begin{tabular}{cccccccccccccc}
\hline Hr & & \multicolumn{1}{c}{ Generation Output } & \multicolumn{1}{c}{ Total } & Fuel & Startup \\
& G1 & G2 & G3 & G4 & G5 & G6 & G7 & G8 & G9 & G10 & Power & Cost & Cost \\
\hline 1 & 455 & 245 & 0 & 0 & 0 & 0 & 0 & 0 & 0 & 0 & 700 & 13,683 & 0 \\
2 & 455 & 295 & 0 & 0 & 0 & 0 & 0 & 0 & 0 & 0 & 750 & 14,554 & 0 \\
3 & 455 & 370 & 0 & 0 & 25 & 0 & 0 & 0 & 0 & 0 & 850 & 16,809 & 900 \\
4 & 455 & 455 & 0 & 0 & 40 & 0 & 0 & 0 & 0 & 0 & 950 & 18,598 & 0 \\
5 & 455 & 390 & 0 & 130 & 25 & 0 & 0 & 0 & 0 & 0 & 1,000 & 20,020 & 560 \\
6 & 455 & 360 & 130 & 130 & 25 & 0 & 0 & 0 & 0 & 0 & 1,100 & 22,387 & 1,100 \\
7 & 455 & 410 & 130 & 130 & 25 & 0 & 0 & 0 & 0 & 0 & 1,150 & 23,262 & 0 \\
8 & 455 & 455 & 130 & 130 & 30 & 0 & 0 & 0 & 0 & 0 & 1,200 & 24,150 & 0 \\
9 & 455 & 455 & 130 & 130 & 85 & 20 & 25 & 0 & 0 & 0 & 1,300 & 27,251 & 860 \\
10 & 455 & 455 & 130 & 130 & 162 & 33 & 25 & 10 & 0 & 0 & 1,400 & 30,058 & 60 \\
11 & 455 & 455 & 130 & 130 & 162 & 73 & 25 & 10 & 10 & 0 & 1,450 & 31,916 & 60 \\
12 & 455 & 455 & 130 & 130 & 162 & 80 & 25 & 43 & 10 & 10 & 1,500 & 33,890 & 60 \\
13 & 455 & 455 & 130 & 130 & 162 & 33 & 25 & 10 & 0 & 0 & 1,400 & 30,058 & 0 \\
14 & 455 & 455 & 130 & 130 & 85 & 20 & 25 & 0 & 0 & 0 & 1,300 & 27,251 & 0 \\
15 & 455 & 455 & 130 & 130 & 30 & 0 & 0 & 0 & 0 & 0 & 1,200 & 24,150 & 0 \\
16 & 455 & 310 & 130 & 130 & 25 & 0 & 0 & 0 & 0 & 0 & 1,050 & 21,514 & 0 \\
17 & 455 & 260 & 130 & 130 & 25 & 0 & 0 & 0 & 0 & 0 & 1,000 & 20,642 & 0 \\
18 & 455 & 360 & 130 & 130 & 25 & 0 & 0 & 0 & 0 & 0 & 1,100 & 22,387 & 0 \\
19 & 455 & 455 & 130 & 130 & 30 & 0 & 0 & 0 & 0 & 0 & 1,200 & 24,150 & 0 \\
20 & 455 & 455 & 130 & 130 & 162 & 33 & 25 & 10 & 0 & 0 & 1,400 & 30,058 & 490 \\
21 & 455 & 455 & 130 & 130 & 85 & 20 & 25 & 0 & 0 & 0 & 1,300 & 27,251 & 0 \\
22 & 455 & 455 & 0 & 0 & 145 & 20 & 25 & 0 & 0 & 0 & 1,100 & 22,736 & 0 \\
23 & 455 & 420 & 0 & 0 & 25 & 0 & 0 & 0 & 0 & 0 & 900 & 17,685 & 0 \\
24 & 455 & 345 & 0 & 0 & 0 & 0 & 0 & 0 & 0 & 0 & 800 & 15,427 & 0 \\
\hline & & & & & & & & & & & &
\end{tabular}


Table 4. Commitment scheduling for 100-unit system achieved by BDE

\begin{tabular}{|c|c|}
\hline $\mathrm{Hr}$ & Unit Schedule \\
\hline 1 & 11111111111111111111100000000000000000000000000000000000000000000000000000000000000000000000000000000000000 \\
\hline 2 & 1111111111111111111110000000000000000000000000000000000000000000000000000000000000000000000000000000 \\
\hline 3 & 111111111111111111111000000000000000000000000000100100000000000000000000000000000000000000000000000000 \\
\hline 4 & 111111111111111111111000000000000000000001110111111100000000000000000000000000000000000000000000000000 \\
\hline 5 & 11111111111111111111000000000000001001100111111111100000000000000000000000000000000000000000000000000000 \\
\hline 6 & 11111111111111111111110000001000111111111111111111111100000000000000000000000000000000000000000000000000000 \\
\hline 7 & 11111111111111111111100010101111111111111111111111100000000000000000000000000000000000000000000000000000 \\
\hline 8 & 111111111111111111111111111111111111111111111111111111100000000000000000000000000000000000000000000000000 \\
\hline 9 & 111111111111111111111111111111111111111111111111111111101110111000101101110000000000000000000000000000000 \\
\hline 10 & 11111111111111111111111111111111111111111111111111111111111111111111111111111111111101011100000000000000000000 \\
\hline 11 & 1111111111111111111111111111111111111111111111111111111111111111111111111111111111111111111110111010110010000000 \\
\hline 12 & 1111111111111111111111111111111111111111111111111111111111111111111111111111111111111111111111111011111101 \\
\hline 13 & 111111111111111111111111111111111111111111111111111111111111111111111111111111100111110100100000000000000000 \\
\hline 14 & 11111111111111111111111111111111111111111111111111111111101111010111010100000000000000000000000000000000000 \\
\hline 15 & 111111111111111111111111111111111111111111111111111111100000000000000000000000000000000000000000000000000 \\
\hline 16 & 111111111111111111111111111111111111111111111111111111100000000000000000000000000000000000000000000000000000 \\
\hline 17 & 1111111111111111111111111111111111111111111111111111000000000000000000000000000000000000000000000000000 \\
\hline 18 & 11111111111111111111111111111111111111111111111111111111110000000000000000000000000000000000000000000000000000 \\
\hline 19 & 11111111111111111111111111111111111111111111111111111111000000000000010100000000000000000000000000000000000 \\
\hline 20 & 1111111111111111111111111111111111111111111111111111111111111111111111111111111011011111000000000100000000000 \\
\hline 21 & 1111111111111111111111010001101111111111111111111111111111111111111111111111000000000000000000000000000000 \\
\hline 22 & 1111111111111111111110000000000110010000001011111011111111111111101011110000000000000000000000000000000 \\
\hline 23 & 111111111111111111111100000000000000000000001011001010000000000000000000000000000000000000000000000000000 \\
\hline 24 & 111111111111111111111100000000000000000000000000000000000000000000000000000000000000000000000000000000 \\
\hline
\end{tabular}

Fig. 5 illustrates the scaling of the execution time of the BDE with the system size. As shown in Fig. 5, the execution time of the BDE increases in a quadratic way with the number of units, and the approximate time of more than 100-unit systems may be predicted from this curve. This implies that the proposed BDE can be applied to practical large-scale UC problems.

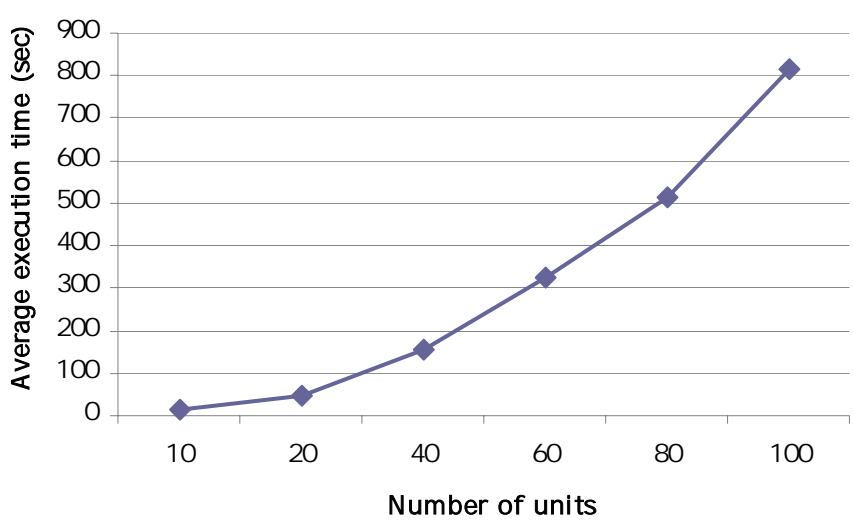

Fig. 5. Scaling of the average execution time of the BDE

\section{Conclusion}

DE is a floating-point encoding evolutionary algorithm for global optimization over continuous spaces and has been successfully applied to a wide range of optimization problems. However, standard DE algorithms have operated in continuous space. It is essential to develop a binary version of DE since many optimization problems, including the UC problem, are set in a space featuring discrete, qualitative distinctions between variables and between levels of variables. In this paper, a new binary differential evolution (BDE) algorithm is proposed for binary optimization problems and is applied to UC problems. In addition, this paper proposes heuristic-based constraint treatment techniques to handle the minimum up/down time and spinning reserve constraints in UC problems. The unit de-commitment approach is also proposed in order to prevent the excessive spinning reserve for cost savings. The proposed BDE was applied to test power systems of up to 100-units along with 24-hour load demands and the results were compared with those of previous works. The simulation results clearly reveal that the proposed BDE algorithm can be used as an excellent optimizer in solving large-scale UC problems.

\section{References}

[1] Wood, A. J., and Wollenberg, B. F., Power Generation, Operation, and Control. New York, John Wiley \& Sons, Inc., 1984.

[2] Burns, R. M., and Gibson, C. A., "Optimization of priority lists for a unit commitment program”, Proc. IEEE Power Engineering Society Summer Meeting, Paper A, 75 453-1, 1975.

[3] Sheble, G. B., "Solution of the unit commitment problem by the method of unit periods", IEEE Trans. on Power Systems, Vol. 5, No. 1, pp. 257-260, Feb. 1990.

[4] Snyder Jr., W. L., Powell Jr., H. D., and Rayburn, J. C., "Dynamic programming approach to unit commitment” IEEE Trans. on Power Apparatus and Systems, Vol. PAS-2, pp. 339-350, May 1987.

[5] Ouyang, Z., and Shahidehpour, S. M., “An intelligent dynamic programming for unit commitment application”, IEEE Trans. on Power Systems, Vol. 6, No. 3, pp. 1203-1209, Aug. 1991.

[6] Merlin, A., and Sandrin, P., "A new method for unit commitment at Electricite de France", IEEE Trans. on Power Apparatus and Systems, Vol. PAS-102, pp. 1218-1255, May 1983.

[7] Zhuang, F., and Galiana, F. D., "Toward a more rigorous and practical unit commitment by Lagrangian relaxation”, IEEE Trans. on Power Systems, Vol. 3, No. 2, pp. 763-770, May 1988.

[8] Cohen, A. I., and Yoshimura, M., "A branch-andbound algorithm for unit commitment”, IEEE Trans. on Power Apparatus and Systems, Vol. PAS-102, pp. 444-451, Feb. 1983.

[9] Muckstadt, J. A., and Wilson, R. C., “An application of mixed-integer programming duality to scheduling thermal generating systems”, IEEE Trans. on Power Apparatus and Systems, pp. 1968-1978, 1968. 
[10] Kazarlis, S. A., Bakirtzis, A. G., and Petridis, V., “A genetic algorithm solution to the unit commitment problem”, IEEE Trans. on Power Systems, Vol. 11, No. 1, pp. 83-92, Feb. 1996.

[11] Swarup, K. S., and Yamashiro, S., "Unit commitment solution methodology using genetic algorithm”, IEEE Trans. on Power Systems, Vol. 17, pp. 87-91, Feb. 2002.

[12] Juste, K. A., Kita, H., Tanaka, E., and Hasegawa, J., "An evolutionary programming solution to the unit commitment problem”, IEEE Trans. on Power Systems, vol. 14, pp. 1452-1459, Nov. 1999.

[13] Chen, H, and Wang, X., "Cooperative coevolutionary algorithm for unit commitment”, IEEE Trans. on Power Systems, vol. 16, pp. 128-133, Feb. 2002.

[14] Zhuang, F., and Galiana, F. D., "Unit commitment by simulated annealing”, IEEE Trans. on Power Systems, Vol. 5, No. 1, pp. 311-317, Feb. 1990.

[15] Simopoulos, D. N., Kavatza, S. D., and Vournas, C. D., "Unit commitment by an enhanced simulated annealing algorithm”, IEEE Trans. on Power Systems, Vol. 21, No. 1, pp. 68-76, Feb. 2006.

[16] Zhao, B., Guo, C. X., Bai, B. R., and Cao, Y. J., “An improved particle swarm optimization algorithm for unit commitment”, Electrical Power \& Energy Systems, Vol. 28, Issue 7, pp. 482-490, Sep. 2006.

[17] Storn, R., and Price, K., "Differential evolution - A simple and efficient heuristic for global optimization over continuous spaces”, Journal of Global Optimization, Vol. 11, pp. 341-359, 1997.

[18] Chang, C. Wong, J., Chiou, J., and Su, C., "Robust searching hybrid differential evolution method for optimal reactive power planning in large-scale distribution systems”, Electric Power Systems Research, pp. 1-8, May 2006.

[19] Arora, J.S., Introduction to Optimum Design, McGrawHill, Inc., 1989.

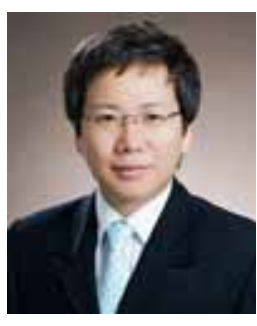

Yun-Won Jeong received his B.S., M.S. and Ph.D. degrees from Konkuk University, Seoul, Korea, in 2002, 2004, and 2007, respectively. Currently, he works in the Business Development Group at POSCO POWER Corporation. His research interests include power system operation and planning, electricity markets, and computational intelligence and their application to power systems.

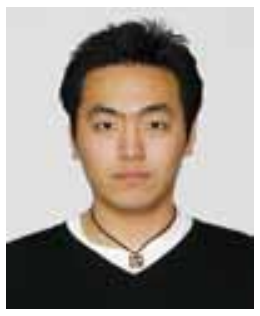

Woo-Nam Lee received his B.S. and M.S. degrees from Konkuk University in 2005 and 2007, respectively. Currently, he is enrolled in a doctoral program at Konkuk University. His major research topics include power system operation and economics.

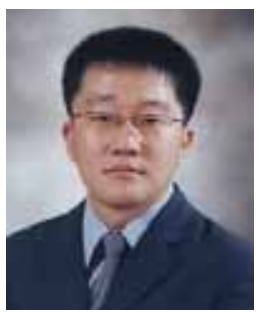

Hyun-Houng Kim received his B.S. and M.S. degrees from Konkuk University in 2004 and 2006, respectively. Currently, he is enrolled in a doctoral program at Konkuk University. His major research topics include power system operation and economics.

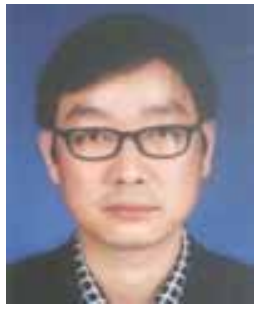

Jong-Bae Park received his B.S., M.S. and Ph.D. degrees from Seoul National University in 1987, 1989, and 1998, respectively. From 1989-1998, he was with Korea Electric Power Corporation, and from 1998-2001 he was an Assistant Professor at Anyang University, Korea. Currently, he is an Associate Professor of Electrical Engineering at Konkuk University, Seoul, Korea. His major research topics include power system operation, planning, economics, and markets.

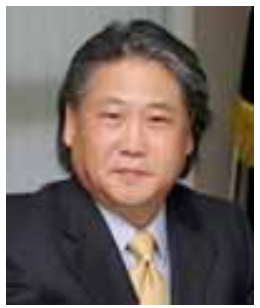

Joong-Rin Shin received his B.S, M.S. and Ph.D. degrees from Seoul National University in 1977, 1984, and 1989, respectively. From 1977-1990, he was with Korea Electric Power Corporation as a researcher. Since 1990, he has been with Konkuk University, Seoul, Korea, where he is currently a Professor of Electrical Engineering. His major research field is in power system operation and planning. 